\title{
Differential Membrane Packing of Stereoisomers of Bis(monoacylglycero)phosphate ${ }^{\dagger}$
}

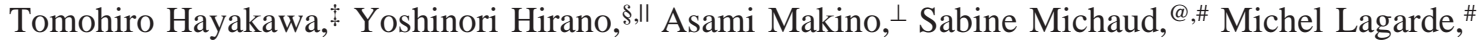 \\ Jean-François Pageaux, ${ }^{\#}$ Alain Doutheau ${ }^{\circledR}$ Kazuki Ito, ${ }^{+}$Tetsuro Fujisawa, ${ }^{+}$Hiroshi Takahashi,,$+\bullet$ and

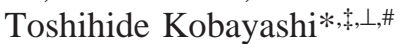 \\ Lipid Biology Laboratory, RIKEN (Institute of Physical and Chemical Research), Computational Astrophysics Laboratory, \\ RIKEN, and Supra-Biomolecular System Research Group, RIKEN Frontier Research System, 2-1, Hirosawa, Wako-shi, Saitama \\ 351-0198, Japan, Laboratoire de Chimie Organique, UMR 5181 CNRS, Université Lyon 1, and Inserm UMR 585, Institut \\ Multidisciplinaire de Biochimie des Lipides (IMBL), Institut National des Sciences Appliquees (INSA)-Lyon, Villeurbanne, \\ F-69621 France, Biometal Science Laboratory, RIKEN SPring-8 Center, Sayo, Hyogo 679-5148, Japan, and \\ Department of Physics, Gunma University, Maebashi 371-8510, Japan
}

Received April 14, 2006; Revised Manuscript Received May 24, 2006

\begin{abstract}
Bis(monoacylglycero)phosphate (BMP) reveals an unusual $s n-1, s n-1^{\prime}$ stereoconfiguration of glycerophosphate. We synthesized $s n$-(3-myristoyl-2-hydroxy)glycerol-1-phospho-sn-1'-(3'-myristoyl-2'hydroxy)glycerol $\left(1,1^{\prime}\right.$-DMBMP) and characterized the thermotropic phase behavior and membrane structure, in comparison with those of the corresponding sn-3:sn-1' stereoisomer (3,1'-DMBMP), by means of differential scanning calorimetry (DSC), small- and wide-angle X-ray scattering (SAXS and WAXS, respectively), pressure-area $(\pi-A)$ isotherms, epifluorescence microscopy of monolayers, and molecular dynamics (MD) simulations. In DSC, these lipids exhibited weakly energetic broad peaks with an onset temperature of $9{ }^{\circ} \mathrm{C}$ for $1,1^{\prime}$-DMBMP and $18{ }^{\circ} \mathrm{C}$ for $3,1^{\prime}$-DMBMP. In addition, a highly cooperative, strongly energetic transition peak was observed at $\sim 40{ }^{\circ} \mathrm{C}$ for $1,1^{\prime}$-DMBMP and $\sim 42{ }^{\circ} \mathrm{C}$ for $3,1^{\prime}$-DMBMP. These results are supported by the observation that 1,1'-DMBMP exhibited a larger phase transition pressure $\left(\pi_{\mathrm{c}}\right)$ than 3,1'-DMBMP. Small- and wide-angle X-ray scattering measurements identified these small and large energetic transitions as a quasi-crystalline $\left(\mathrm{L}_{\mathrm{c} 1}\right)$ - quasi-crystalline with different tilt angle $\left(\mathrm{L}_{\mathrm{c} 2}\right)$ phase transition and an $\mathrm{L}_{\mathrm{c} 2}-\mathrm{L}_{\alpha}$ main phase transition, respectively. X-ray measurements also revealed that these DMBMPs undergo an unbinding at the main phase transition temperature. The MD simulations estimated stronger hydrogen bonding formation in the 3,1'-DMBMP membrane than in 1,1'-DMBMP, supporting the experimental data.
\end{abstract}

Bis(monoacylglycero)phosphate, also known as lysobisphosphatidic acid (LBPA), was first described in pig and rabbit pulmonary alveolar macrophages by Body and Gray (1). BMPs comprise less than $1 \%$ of the total phospholipids in most mammalian cells $(2,3)$. However, the content is increased to $15 \%$ in late endosomes (4). In the specific internal membrane domains of this organelle, this lipid comprises more than $70 \%$ of the total phospholipids (5).

\footnotetext{
$\doteqdot$ This work was supported by grants from the Ministry of Education, Science, Sports and Culture of Japan, grants from the RIKEN Frontier Research System, and the Bioarchitect Project of RIKEN to T.K. Y.H. was a special postdoctoral fellow of RIKEN.

* To whom correspondence should be addressed: Lipid Biology Laboratory, RIKEN (Institute of Physical and Chemical Research), 2-1, Hirosawa, Wako-shi, Saitama 351-0198, Japan. Telephone: +81-48467-9612. Fax: +81-48-467-8693. E-mail: kobayasi@riken.jp.

Lipid Biology Laboratory, RIKEN.

$\S$ Computational Astrophysics Laboratory, RIKEN.

"Present address: Department of Pharmacology, Keio University School of Medicine, 35 Shinanomachi, Shinjuku-ku, Tokyo 160-8582, Japan.

${ }^{\perp}$ Supra-Biomolecular System Research Group, RIKEN Frontier Research System.

@ UMR 5181 CNRS.

\# Inserm UMR 585.

+ RIKEN SPring-8 Center.

- Gunma University.
}

Because of the high level of accumulation in late endosomes, BMP is assumed to be important for the structural and functional integrity of the organelle. Indeed, a series of experiments has demonstrated that agents that perturb BMP induce alterations in the sorting and trafficking of proteins and/or the receptor in late endosomes accompanied by structural changes of the late edonsome organelle $(4,6)$ along with abnormal accumulation of cholesterol (7). Moreover, BMP is found to be essential for the activator-stimulated hydrolysis of ganglioside $\mathrm{G}_{\mathrm{M} 1}(8)$ and $\mathrm{G}_{\mathrm{M} 2}(9)$ and for the hydrolysis of ceramide by acid ceramidase (10).

BMP has unique structural characteristics with various fatty acid compositions depending on the source. Whereas all other mammalian phospholipids display a $s n$-3-glycerophosphate stereoconfiguration, BMP possesses an $s n-1: s n$ $1^{\prime}$ structure and one acyl group attached to each of the glycerol moieties $(11-13)$. An $s n-3: s n-1^{\prime}$ isomer has also been reported for BMP in BHK cells (14). However, the $s n-3: s n-1^{\prime}$ isomer in mammalian cells is postulated to be an obligatory intermediate in the synthesis of $s n-1: s n-1^{\prime}-\mathrm{BMP}$ from $s n-3: s n-1^{\prime}-\mathrm{PG}(15-21)$. The physiological significance of the $s n-1: s n-1^{\prime}$ stereoconfiguration is not well understood. $s n-1$ phospholipids are resistant to most phospholipases. 
However, recent work has revealed the existence of phospholipase $A_{2}$ that catalyzes the hydrolysis of naturally occurring BMP (22). Considering the high level of accumulation of BMP in late endosomes, it is suggested that the specific stereoconfiguration of BMP plays an important role in the function of degradative organelles which are forming characteristic multilamellar/multivesicular structures.

To elucidate the functional role of BMP in late endosomes, determination of the biophysical characteristics of this lipid is a prerequisite. Such biophysical studies have just started recently. Holopainen et al. investigated the membrane properties of dioleoyl-BMP (DOBMP) by means of Langmuir-lipid monolayers, electron microscopy, differential scanning calorimetry (DSC), and fluorescence spectroscopy (23). They found DOBMP forms multilamellar vesicles with no structural transitions or phase transitions between 10 and $80{ }^{\circ} \mathrm{C}$ over a $\mathrm{pH}$ range of $3.0-7.4$. They also found no evidence of lateral phase separation in both DOBMP/DPPC and DOBMP/POPC mixtures in $5 \mathrm{mM}$ HEPES and $0.1 \mathrm{mM}$ EDTA ( $\mathrm{pH}$ 7.4). Matsuo et al. found that BMP can induce the formation of multivesicular liposomes that resemble the multivesicular endosomes where this lipid is found in vivo. They also reported that the formation of the multivesicular liposomes depends on the acyl chain composition of BMP and the position of esterification in the glycerophosphate backbone (24). We recently reported that DOBMP membranes form a closely packed multilamellar structure at low $\mathrm{pH}$ by adding the hydrophobic amine, D-threo-1-phenyl-2decanoylamino-3-morpholino-1-propanol (D-PDMP) (25). We also showed that this interaction between BMP and D-PDMP is a major cause of the structural alteration of the degradative organelles found in D-PDMP-treated cells and the accumulation of cholesterol in these organelles.

The goal of this study is to characterize the thermotropic and structural behavior of chemically synthesized $1,1^{\prime}$ DMBMP and to compare the obtained results with those of the $s n-3: s n-1^{\prime}$ isomer. We employed the established techniques of DSC, X-ray scattering, $\pi-A$ isotherms, epifluorescence microscopy of lipid monolayers, and MD simulations. Although naturally occurring BMP in mammals favors the $s n-1: s n-1^{\prime}$ headgroup configuration rather than the $s n$ $3: s n-1^{\prime}$ configuration, the similarities or differences in the physicochemical properties between these two headgroup structures have not been described. The acyl chain location of natural BMPs, namely, at $s n-2\left(2^{\prime}\right)$ or $s n-3\left(3^{\prime}\right)$ positions, has not been established. However, a recent study has suggested that BMPs prefer $s n-2: s n-2^{\prime}$ acyl chain positions (5). In this study, to focus on the effects of headgroup configuration on the thermotropic phase behaviors of BMP, thermodynamically stable 3,3'- and 1,3'-diacyl BMPs were employed since 2,2'-diacyl BMP is quite unstable due to 2,3acyl migration. Although the lipid containing two saturated fatty acids is a minor molecular species in naturally occurring BMP, DMBMP enables us to compare the stereoisomers of BMP using various physicochemical techniques at physiological temperature. Here we show that $3,1^{\prime}$-DMBMP packs more tightly than $1,1^{\prime}$ isomer. These findings are discussed in relation to the effect of the stereoconfiguration on the molecular packing of these lipids, particularly on intermolecular hydrogen bonding and hydration in the interfacial region of the BMP bilayer.

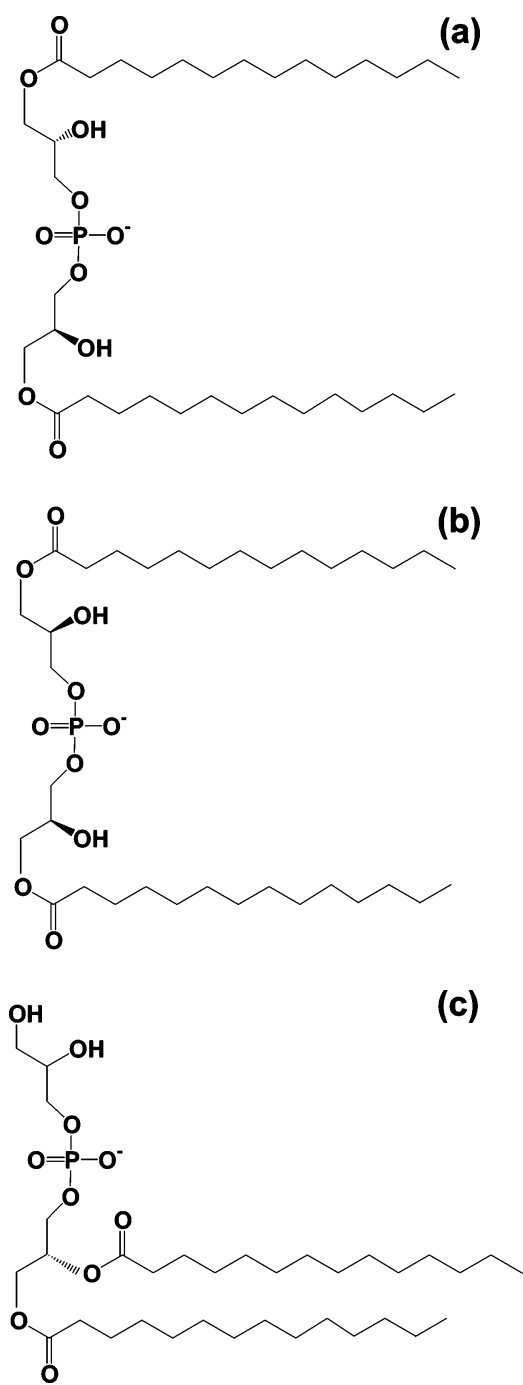

FIGURE 1: Structure of BMP employed in this study: (a) 3,3'dimyristoyl-sn-1,1'-BMP (1,1'-DMBMP) and (b) 1,3'-dimyristoyl$s n$-3, 1'-BMP (3,1'-DMBMP). 1,2-Dimyristoyl-sn-glycero-3-phospho$1^{\prime}$-glycerol, a structural isomer of BMP, is shown in panel $\mathrm{c}$.

\section{MATERIALS AND METHODS}

\section{Materials}

$s n$-(3-Myristoyl-2-hydroxy)glycerol-1-phospho-sn-1'-(3'myristoyl-2'-hydroxy)glycerol (1,1'-DMBMP) (Figure 1) was prepared according to the published method (26) with a slight modification in the protection of $(S, S)$-2-(diphenylmethylsilyl)ethylbis(sn-glycerol)phosphate. The primary alcohol of this compound was protected as tert-butyldiphenylsilyl ethers using the preformed silylating reagent (27). Deprotection in the final step of synthesis with tetrabutylammonium fluoride (TBAF) in tetrahydrofuran (THF) was accompanied by a simultaneous migration of the acyl groups from the $s n-2$ to $s n-3$ position, giving rise to $3,3^{\prime}$-dimyristoyl-sn-1,1'-BMP (26).

1,3'-Dimyristoyl-sn-3,1'-BMP (3,1'-DMBMP), 1,2-dipalmitoyl-sn-glycero-3-phospho-rac-1-glycerol (DPPG), and 1,2-dimyristoyl-sn-glycero-3-phosphoethanolamine (DMPE) were purchased from Avanti Polar Lipids, Inc. (Alabaster, AL). The purity of the lipids was checked by thin-layer chromatography (TLC), and only one spot was detected on the TLC plate (Kieselgel 60, Merck). Lipids were quantified with the method of Rouser et al. (2). 2-(4,4-Difluoro-5,7- 
dimethyl-4-bora-3a,4a-diaza- $s$-indacene-3-dodecanoyl)-1hexadecanoyl-sn-glycero-3-phosphocholine (Bodipy- $\mathrm{C}_{12}-\mathrm{PC}$ ) was purchased from Molecular Probes (Eugene, OR).

\section{Differential Scanning Calorimetry (DSC)}

BMP samples were prepared for DSC as follows. A lipid film was formed from a chloroform solution of lipids under a stream of nitrogen gas, dried under high vacuum overnight, hydrated, and vortexed with a buffer solution containing 20 mM 4-(2-hydroxyethyl)-1-piperizineethanesulfonic acid (HEPES), $100 \mathrm{mM} \mathrm{NaCl}$, and $10 \mathrm{mM}$ EDTA (pH 7.0) or 20 mM 2-( $N$-morpholino)ethanesulfonic acid (MES), $100 \mathrm{mM}$ $\mathrm{NaCl}$, and $10 \mathrm{mM}$ EDTA ( $\mathrm{pH}$ 5.3). The final concentration of BMP was $0.5 \mathrm{mM}$. DSC studies were performed with a MicroCal (Northampton, MA) VP-DSC microcalorimeter. DSC thermograms were recorded at a scan rate of $10^{\circ} \mathrm{C} / \mathrm{h}$. Sample damage was checked after measurement by using TLC. No trace of lyso compound or fatty acid was found under these conditions. The obtained data were plotted and analyzed with Origin (OriginLab Corp., Northampton, MA).

\section{Small- and Wide-Angle X-ray Scattering}

The dispersed BMP samples for X-ray measurements were prepared essentially as described for DSC measurements using $5 \mathrm{mM}$ lipids. SAXS and WAXS measurements were carried out at RIKEN Structural Biology Beamline I (BL45XU) (28) at SPring-8, $8 \mathrm{GeV}$ synchrotron radiation source (Hyogo, Japan). The X-ray wavelength that was used was $0.9 \AA$, and the beam size at the sample position was $\sim 0.4 \mathrm{~mm} \times 0.7 \mathrm{~mm}$. The sample-to-detector distance was $600 \mathrm{~mm}$. Samples were measured in a sample cell with a path length of $1.5 \mathrm{~mm}$ and a pair of thin quartz windows (thickness of $30 \mu \mathrm{m}$ ). The sample temperature was controlled to $\pm 0.01{ }^{\circ} \mathrm{C}$ with a high-precision thermoelectric device. The temperature of samples was maintained for 5-6 min to be equilibrated with each temperature. To minimize the sample damage during observation, three sets of samples were prepared. The first sample was used to measure at 5, 10, 15, and $20^{\circ} \mathrm{C}$, the second sample at 20,25 , and $35^{\circ} \mathrm{C}$, and the third sample at 35,40 , and $45^{\circ} \mathrm{C}$. There were not significant differences in the diffraction patterns between the first and second samples and between the second and third sample measured at the same temperature, indicating that the sample was not significantly damaged under these conditions (29, 30). Buffer profiles were also measured at respective temperatures to subtract the background. The SAXS and WAXS patterns were simultaneously recorded with the RIGAKU imaging plate detector (R-AXIS $\mathrm{IV}^{++}$, active area size of $30 \mathrm{~cm} \times 30 \mathrm{~cm})$. (31). The two-dimensional powder diffraction patterns were circularly averaged and reduced to one-dimensional profiles using FIT2D version 12.012 (http:// www.esrf.fr/computing/scientific/FIT2D/), a two-dimensional data reduction and analysis program. The reciprocal spacings $(s)[s=1 / d=(2 / \lambda) \sin \theta$ (where $d$ is the lattice spacing, $2 \theta$ is the scattering angle, and $\lambda$ is the $\mathrm{X}$-ray wavelength)] were calibrated with silver behenate by the long-period spacing of $5.838 \mathrm{~nm}(32)$.

\section{Electron Density Distribution of BMP Membranes}

From the obtained SAXS data, one-dimensional electron density profiles, $\rho(z)$, were calculated as

$$
\rho(z)=\sum_{h=1}^{N} f(h)|F(h)| \cos (2 \pi h z / d)
$$

where $h$ is the diffraction order, $f(h)$ the phase factor which must be 1 or -1 for symmetric bilayers, $|F(h)|$ the absolute value of the structure factor, and $z$ the distance from the center of the bilayer. For diffraction patterns from unoriented samples, the structure factor $|F(h)|$ is set equal to $\left[h^{2} I(h)\right]^{1 / 2}$ $(33,34)$, where $I(h)$ is the integrated intensity of the $h$ th order peak. From the obtained four lamellar peaks (first to fourth order) in the small-angle region, 16 possible electron density profiles were all calculated. To choose the most probable candidate from the 16 profiles, it is assumed that the $1,1^{\prime}$ DMBMP bilayer has the highest electron density at the phosphate group at the hydrophilic-hydrophobic interface and has the lowest electron density at the center of the bilayer due to the localization of the methyl group of the acyl chains. Four profiles were selected with the assumptions given above, and two of them were ruled out due to the improper electron density profile in the hydrophobic region. Finally, the phase factors, $f(h)$ in the formula given above, for the first- to fourth-order lamellar diffractions were determined to be $-1,-1,1$, and -1 , respectively.

\section{Pressure-Area Compression Isotherms}

All lipids were dried under high vacuum for more than $24 \mathrm{~h}$ and were carefully quantified by weight using an UMX2 ultra-micro balance (Mettler-Toledo Inc., Columbus, $\mathrm{OH}$ ). Surface pressure-molecular area $(\pi-A)$ isotherms were measured using a computer-controlled, Langmuir-type film balance (USI System, Fukuoka, Japan), calibrated according to the equilibrium spreading pressures of stearic acid. Lipids, dissolved in chloroform, were spread onto $20 \mathrm{mM}$ HEPES buffer [containing $100 \mathrm{mM} \mathrm{NaCl}$ and $10 \mathrm{mM}$ EDTA $(\mathrm{pH}$ 7.0)] subphase. Lipid films were compressed at a rate of 0.1 $\mathrm{nm}^{2}$ per molecule per minute after an initial delay period of 30 min. All experiments were carried out at room temperature, and the subphase temperature was monitored (23.5 \pm $\left.0.5^{\circ} \mathrm{C}\right)$.

\section{Lipid Monolayers and Epifluorescence Microscopy}

Bodipy- $\mathrm{C}_{12}$-PC-containing lipid monolayers (1 mol \% Bodipy- $\mathrm{C}_{12}$-PC) were obtained by spreading $\sim 20 \mu \mathrm{L}$ of a lipid solution in chloroform over a subphase of $20 \mathrm{mM}$ HEPES, $100 \mathrm{mM} \mathrm{NaCl}$, and $10 \mathrm{mM}$ EDTA (pH 7.0). After solvent evaporation, the monolayer was compressed at a rate of $0.25 \mathrm{~nm}^{2}$ per molecule per minute to the desired surface pressure $(\pi=5-15 \mathrm{mN} / \mathrm{m})$. Epifluorescence microscopy was carried out using an Olympus Power BX fluorescence microscope equipped with an LM Plan Fl 50× objective and a Toshiba 3CCD camera. All experiments were carried out with a subphase temperature of $23.5 \pm 0.5^{\circ} \mathrm{C}$.

\section{Molecular Dynamics (MD) Simulations}

Atomic Parameters. We used all-atom models of $1,1^{\prime}-$ and 3,1'-DMBMPs for MD simulations. The charge distributions on both lipids were calculated using Gaussian 03 program (35). The atomic charges were determined by the secondorder Møller-Plesset correlation (MP2) level with the $6-31 \mathrm{G}^{* *}$ basis set, and restrained electrostatic potential 
(RESP) fitting was used. Before determining the atomic charges for both lipids, we carried out the energy optimization calculations at the PM3 level to construct their conformations. To calculate other interactions (bond stretching, bending, torsion, and van der Waals), the AMBER parameter set was used (36).

Simulation Conditions. Both 1,1'- and 3,1'-DMBMPs bilayer systems consisted of $256(16 \times 8 \times 2)$. The initial size of the bilayer was $7.2 \mathrm{~nm} \times 12.8 \mathrm{~nm} \times 4.6 \mathrm{~nm}$. To construct the initial bilayer structures of DMBMPs, the acyl chains of each lipid were placed on the hexagonal on the $x$ and $y$ planes to fit the experimental data and the end of the acyl chains overlapped one another on the $z$ plane. The bilayer was immersed in a $7.2 \mathrm{~nm} \times 13.1 \mathrm{~nm} \times 7.1 \mathrm{~nm}$ box filled with TIP3P water. Water molecules whose oxygen atoms were closer than $0.3 \mathrm{~nm}$ to any DMBMP atoms were removed. The total number of water molecules was $\sim 6000$, and 256 sodium ions were generated for the system to be neutral. Approximately $0.5 \mathrm{~nm}$ thick water layers ware formed on both sides of the lipid bilayer of the membrane. MD simulations were carried out at a constant number of molecules, a pressure of $1 \mathrm{~atm}$, and a temperature of $278 \mathrm{~K}$ $\left(5^{\circ} \mathrm{C}\right)$ (NPT ensemble) after each system had been heated to $278 \mathrm{~K}$ over the first $100 \mathrm{ps}$. The time step was set at $1 \mathrm{fs}$. The bond lengths involving hydrogen atoms were constrained to equilibrium lengths using the SHAKE method. The data were collected through a series of additional $3.0 \mathrm{~ns}$ MD simulations performed at a constant temperature of $278 \mathrm{~K}$ according to Berendsen's algorithm with a coupling time of 0.2 ps. The particle mesh Ewald (PME) method direct space cutoff distance is $1.2 \mathrm{~nm}$. The program used for these MD simulations was Amber 8.0 (36).

\section{RESULTS AND DISCUSSION}

Differential Scanning Calorimetry (DSC). To investigate the thermotropic phase behavior of DMBMPs, DSC measurements were performed. Panels a-d of Figure 2 show the DSC thermograms of $1,1^{\prime}$ - and 3,1'-DMBMP at $\mathrm{pH} 7.0$ and 5.3. For both of the DMBMPs, when heated, two distinct thermotropic phase transitions were observed under both $\mathrm{pH}$ conditions. One of these is the lower-temperature transition detected between 10 and $20{ }^{\circ} \mathrm{C}$ for $1,1^{\prime}$-DMBMP and between 20 and $35{ }^{\circ} \mathrm{C}$ for $3,1^{\prime}$-DMBMP. Another is the higher-temperature transition centered at $\sim 40{ }^{\circ} \mathrm{C}$ for $1,1^{\prime}$ DMBMP and $\sim 42{ }^{\circ} \mathrm{C}$ for $3,1^{\prime}$-DMBMP. For both DMBMPs, the lower-temperature transitions were fairly broad and contained multiple peaks, while the higher-temperature transitions were strongly energetic $(12-14 \mathrm{kcal} / \mathrm{mol})$. In both the lower- and higher-temperature transitions, the transition temperatures $\left(T_{\mathrm{m}}\right)$ of $3,1^{\prime}$-DMBMP were higher than those of 1,1'-DMBMP (see Table 1). For both DMBMPs, when cooled, the peak of the higher-temperature transition observed on heating was split into multiple peaks and the lowertemperature transition exhibited some cooling hysteresis. Although slight differences were observed in the onset temperature of the transitions and in peak broadening, the overall profile of the thermograms (Figure 2) and calorimetric parameters (Table 1) are very similar at both $\mathrm{pH} 7.0$ and 5.3, suggesting that these DMBMPs undergo the same structural alterations at the phase transitions in this physiological $\mathrm{pH}$ range.
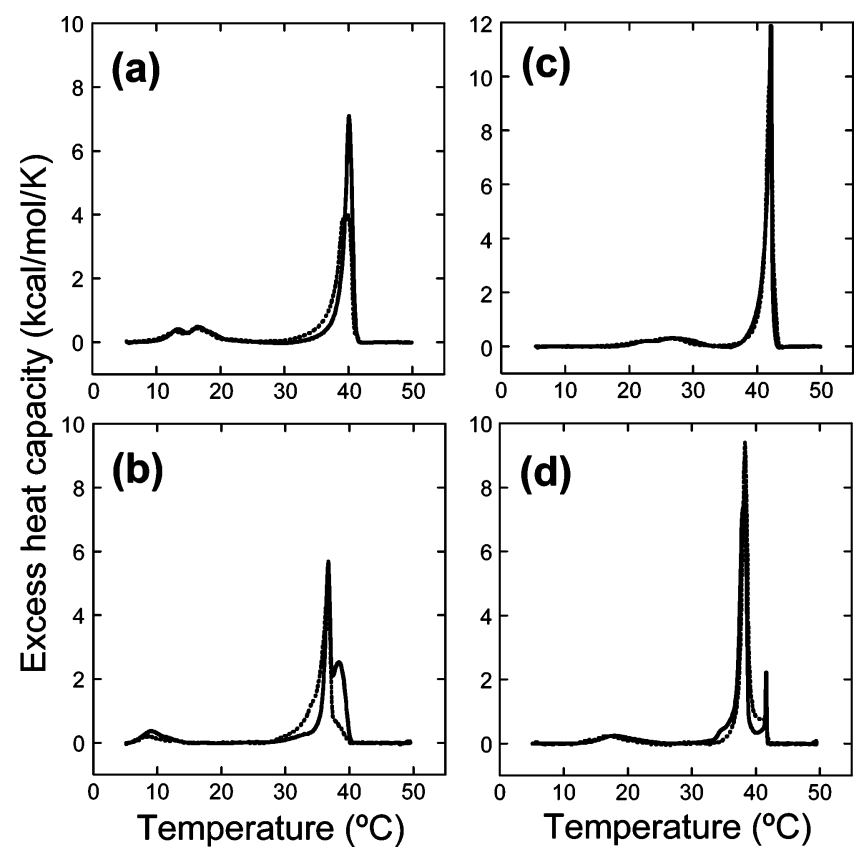

FIGURE 2: Heating ( $\mathrm{a}$ and $\mathrm{c}$ ) and cooling ( $\mathrm{b}$ and $\mathrm{d}$ ) DSC thermograms exhibited by $1,1^{\prime}-$ and $3,1^{\prime}$-DMBMP at $\mathrm{pH} 7.0(-)$ and $5.3(\cdots)$ : (a and b) 1,1'-DMBMP and (c and d) 3,1'-BMP. The thermograms were the second scan recorded at a scan rate of $10{ }^{\circ} \mathrm{C} / \mathrm{h}$.

Table 1: Phase Transition Temperatures $\left(T_{\mathrm{m}}\right)$ and Enthalpies $(\Delta H)$ for DMBMPs ${ }^{a}$

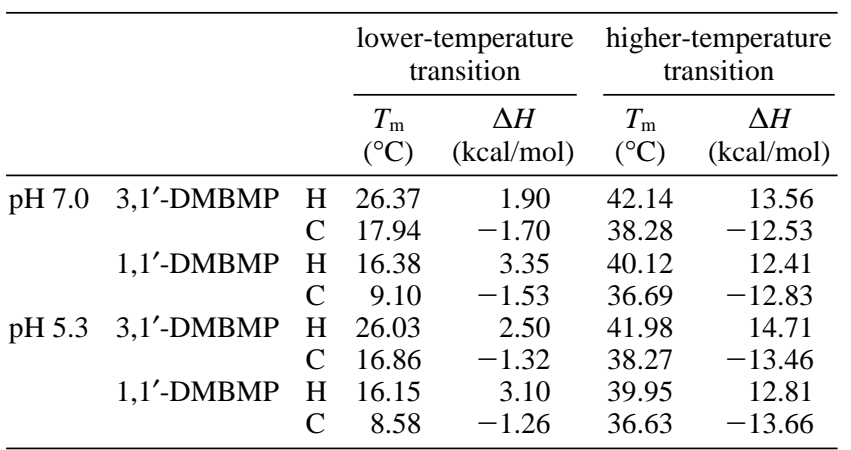

${ }^{a}$ Phase transition temperatures and enthalpies observed at a second heating $(\mathrm{H})$-cooling $(\mathrm{C})$ scan. Lower- and higher-temperature transitions were observed at $\sim 10-35$ and $\sim 35-43{ }^{\circ} \mathrm{C}$, respectively.

Although it was not possible to determine the phases of DMBMPs at temperatures below and above the calorimetric transitions by these DSC thermograms, the appearance of multiple peaks on cooling suggests that these are not simple gel to liquid crystalline transitions. Furthermore, the observed high-temperature transition with a large enthalpy (12-14 $\mathrm{kcal} / \mathrm{mol}$ ) strongly suggests that these are transitions of hydrocarbon chains from a highly ordered crystal or crystallike $\left(\mathrm{L}_{\mathrm{c}}\right)$ state to a disordered melt state $\left(\mathrm{L}_{\alpha}\right)$. Under similar conditions, DMPG and dimyristoylphosphatidylcholine (DMPC) generally show a simple gel $\left(\mathrm{L}_{\alpha}\right)$ to liquid crystalline $\left(\mathrm{L}_{\alpha}\right)$ transition at $\sim 23{ }^{\circ} \mathrm{C}$ with a $5-7 \mathrm{kcal} / \mathrm{mol}$ transition enthalpy (37-42). Considering these values for DMPC and DMPG, it seems likely that the high transition temperature and the large enthalpy observed for DMBMPs are attributed to the attractive interactions between the DMBMP headgroups. Under certain specific conditions, it is reported that DMPG also exhibits such highly energetic transitions. When DMPG is stored for 1 day to 3 months at $0-5^{\circ} \mathrm{C}$, this lipid 

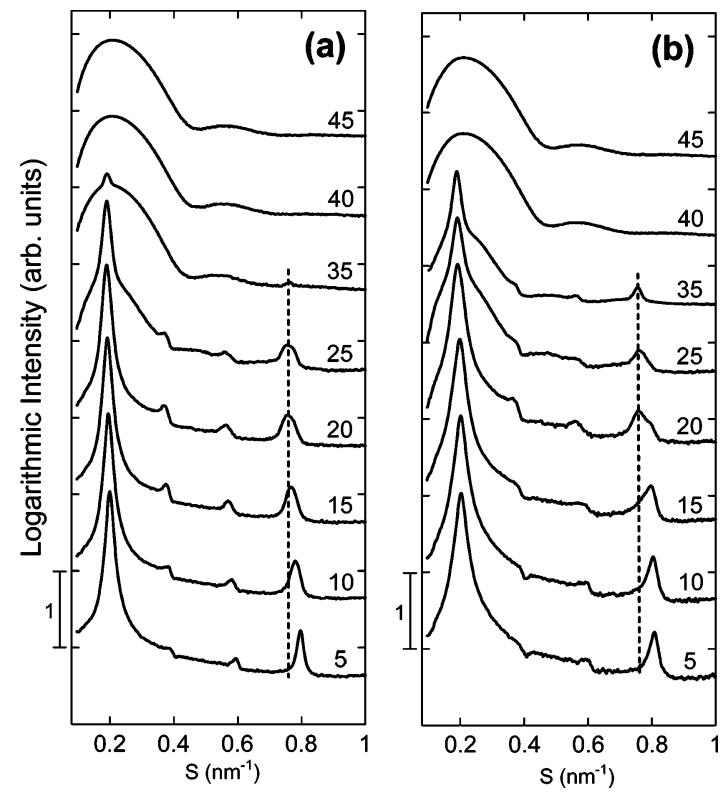

FIGURE 3: Temperature dependence of small-angle X-ray scattering profiles of aqueous dispersions of (a) 1,1'- and (b) 3,1'-DMBMP at $\mathrm{pH}$ 7.0. The numbers indicate the temperature of the sample. Linear dashed lines in panels a and b at $S=0.76 \mathrm{~nm}^{-1}$ are shown to help in the understanding of the peak shift with an increase in temperature.

exhibits large transition enthalpies $(14-19 \mathrm{kcal} / \mathrm{mol})$ at the main phase transition at $\sim 40-42{ }^{\circ} \mathrm{C}$, on the DSC first heating scan $(43-48)$. The authors suggest that the incubation at low temperatures induces DMPG to form a quasicrystalline/crystal-like $\left(\mathrm{L}_{\mathrm{c}}\right)$ phase that involves an intermolecular hydrogen bonding $(43,44,47)$. In the DSC study presented here, we reproducibly observed the transition with a large enthalpy for both DMBMPs without low-temperature storage before the measurements. Together with the result for DMPG, it is conceivable that the intermolecular hydrogen bonding is the major attractive force between the headgroups.

The transition temperature and enthalpy of 3,1'-DMBMP were higher than those of $1,1^{\prime}$-DMBMP. Such stereoconfiguration dependence of the thermotropic parameters has also been reported for DMPG $(48-50)$. In these reports, the transition temperature of DMPG with naturally occurring stereoconfiguration 1,2-dimyristoyl-sn-glycero-3-phosphatidyl-sn-1'-glycerol (1'-DMPG) was lower than that of the corresponding 1,2-dimyristoyl-sn-glycero-3-phosphatidyl-sn$3^{\prime}$-glycerol (3'-DMPG), in the presence of $\mathrm{Na}^{+}$. The higher affinity of $3^{\prime}$-DMPG for $\mathrm{Na}^{+}$is suggested to be due to a slightly different favored conformation of the headgroup glycerol moiety. To elucidate the phase behavior of 3,1'and $1,1^{\prime}$-DMBMP structurally, we next performed X-ray measurements.

Small- and Wide-Angle X-ray Scattering. We employed small- and wide-angle X-ray scattering measurements to investigate and assign the phases of $1,1^{\prime}-$ and $3,1^{\prime}$-DMBMPs at temperatures below and above the calorimetric transitions. Small- and wide-angle scattering patterns were collected simultaneously under the optical conditions described in Materials and Methods. Figure 3 shows the SAXS patterns of $1,1^{\prime}$ - and 3,1'-DMBMP when heated at $\mathrm{pH}$ 7.0. For both DMBMPs, four diffraction peaks fitted a $d, d / 2, d / 3$, and $\mathrm{d} / 4$ series, indicating that they were in lamellar structures. The lamellar repeat distance at $5{ }^{\circ} \mathrm{C}$ was $5.018 \mathrm{~nm}$ for $1,1^{\prime}$ -

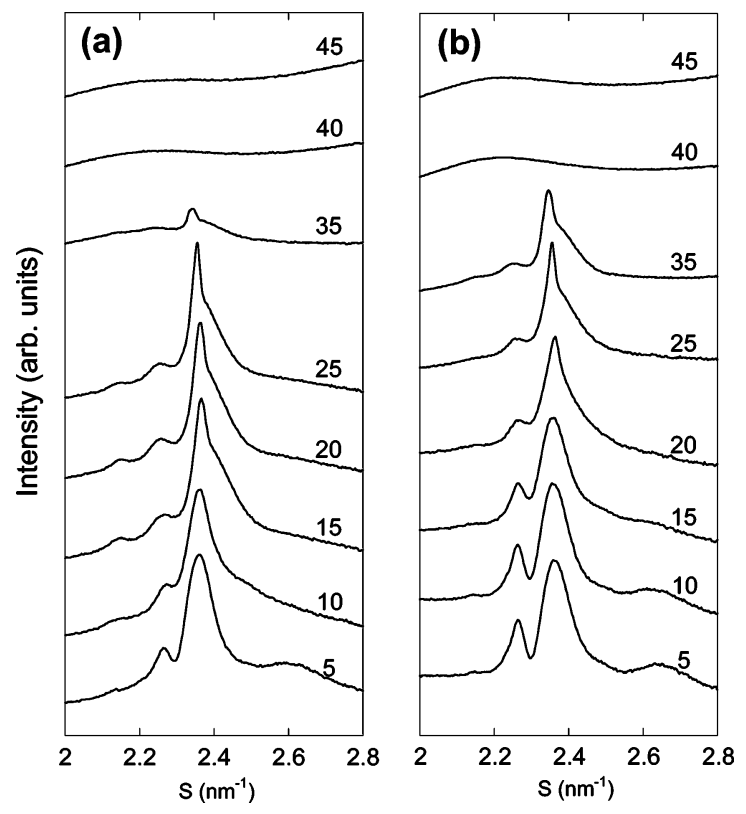

FIGURE 4: Temperature dependence of wide-angle X-ray scattering profiles of aqueous dispersions of (a) $1,1^{\prime}-$ and (b) 3,1'-DMBMP at $\mathrm{pH}$ 7.0. The numbers indicate the temperature of the sample.

DMBMP and $4.935 \mathrm{~nm}$ for 3,1'-DMBMP. These distances became slightly larger with an increase in temperature and reached a value of $\sim 5.2 \mathrm{~nm}$ at $15^{\circ} \mathrm{C}$ for $1,1^{\prime}$-DMBMP and at $25^{\circ} \mathrm{C}$ for $3,1^{\prime}$-DMBMP. When the temperature came close to the phase transition at $\sim 40{ }^{\circ} \mathrm{C}$, a broadening of the lamellar peaks was observed for both DMBMPs, and above the transition temperature, the lamellar peaks all disappeared and only the scattering pattern from the bilayer form factor was observed. The temperature dependency of the scattering patterns and the lamellar distances was almost the same between $\mathrm{pH} 7.0$ and 5.3 (data not shown), indicating that the membrane structures and phase behavior of DMBMPs were not altered in this $\mathrm{pH}$ range.

The packing information with respect to the acyl chains was obtained from WAXS data. Figure 4 shows the WAXS patterns of 1,1'- and 1,3'-DMBMP on heating at $\mathrm{pH}$ 7.0. Like SAXS data (Figure 3), the WAXS profiles of both DMBMPs were similar and not dependent on $\mathrm{pH}$. The appearance of several peaks in the WAXS region is indicative of the formation of an $\mathrm{L}_{\mathrm{c}}$-like or quasi-crystalline phase (termed $\mathrm{L}_{\mathrm{c} 1}$ in this paper) which has a highly ordered chain lattice. A similar WAXS pattern was reported for the quasicrystalline $\mathrm{L}_{\mathrm{c}}$ phase of DMPG, which was stored at $4{ }^{\circ} \mathrm{C}$ for 7 days (43). The peaks were located at $(0.468 \mathrm{~nm})^{-1},(0.441$ $\mathrm{nm})^{-1},(0.424 \mathrm{~nm})^{-1}$, and $(0.385 \mathrm{~nm})^{-1}$ for $1,1^{\prime}$-DMBMP and $(0.465 \mathrm{~nm})^{-1},(0.442 \mathrm{~nm})^{-1},(0.424 \mathrm{~nm})^{-1}$, and $(0.378$ $\mathrm{nm})^{-1}$ for $3,1^{\prime}$-DMBMP at $5^{\circ} \mathrm{C}$ and $\mathrm{pH}$ 7.0. These peaks do not correspond to the higher orders of the lamellar diffractions. These WAXS patterns of DMBMPs were also observed in DPPG in the $\mathrm{L}_{c}$ phase (51-53). However, DPPG in $\mathrm{L}_{\mathrm{c}}$ exhibits a characteristic peak at $\sim(0.78 \mathrm{~nm})^{-1}$ which is missing in WAXS of DMBMPs. Therefore, the highly ordered phase of DMBMPs is not identical to the $\mathrm{L}_{c}$ phase of DPPG. The peak at $\sim(0.38 \mathrm{~nm})^{-1}$ was shifted to a smallangle region $\left[\sim(0.41 \mathrm{~nm})^{-1}\right]$ at $10^{\circ} \mathrm{C}$ for $1,1^{\prime}$-DMBMP and $20^{\circ} \mathrm{C}$ for $3,1^{\prime}$-DMBMP, while the peak at $(0.424 \mathrm{~nm})^{-1}$ became sharper with an increase in temperature. The appearance of the $\sim(0.424 \mathrm{~nm})^{-1}$ peak with such a shoulder 
at the wide-angle region from $\sim(0.38 \mathrm{~nm})^{-1}$ to $(0.41 \mathrm{~nm})^{-1}$ indicates that the chains are packed in an orthorhombic lattice and are tilted with respect to the bilayer normal orientation in the gel phase $(54,55)$. A comparison of the peak position around $\sim(0.38 \mathrm{~nm})^{-1}$ between the two isomers [3,1'DMBMP indicates a spacing, $(0.378 \mathrm{~nm})^{-1}$, that is smaller than that of 1,1'-DMBMP, $(0.385 \mathrm{~nm})^{-1}$ ] suggests the hydrocarbon chains of 3,1'-DMBMP are more tilted than those of $1,1^{\prime}$-DMBMP. This corresponds to the smaller lamellar distance observed in the SAXS measurement for $3,1^{\prime}$-DMBMP at $5{ }^{\circ} \mathrm{C}$ (Figure 3). From the SAXS and WAXS results, it is suggested that the weakly energetic thermotropic transitions around $10-30{ }^{\circ} \mathrm{C}$ observed in the DSC experiment may reflect the structural change of the tilt angle of the lipids. The peak at $\sim(0.44 \mathrm{~nm})^{-1}$, characteristic of the $\mathrm{L}_{\mathrm{c}}$ phase $(52-54)$, was observed until the sample reached the transition temperature at $\sim 32-43{ }^{\circ} \mathrm{C}$; therefore, we have assigned this as another $\mathrm{L}_{\mathrm{c}}$-like highly ordered phase (termed $\mathrm{L}_{\mathrm{c} 2}$ in this paper). Above the transition temperature at $\sim 32-$ $43{ }^{\circ} \mathrm{C}$, peaks in the wide-angle region all disappeared and a broad peak centered at $(0.45 \mathrm{~nm})^{-1}$, characteristic of the fluid liquid crystalline phase $\left(\mathrm{L}_{\alpha}\right)$, was observed for both DMBMPs.

From the observations by small- and wide-angle X-ray scattering together with the DSC measurements, we are able to assign the phases at temperatures below and above the transitions. When heated, 1, $1^{\prime}$-DMBMP undergoes an $\mathrm{L}_{\mathrm{c} 1} \rightarrow$ $\mathrm{L}_{\mathrm{c} 2}$ phase transition centered at $\sim 17{ }^{\circ} \mathrm{C}$ and an $\mathrm{L}_{\mathrm{c} 2} \rightarrow \mathrm{L}_{\alpha}$ main phase transition at $\sim 40{ }^{\circ} \mathrm{C}$, whereas $3,1^{\prime}$-DMBMP undergoes the same phase transitions at $\sim 28$ and $\sim 42{ }^{\circ} \mathrm{C}$, respectively. DSC thermograms revealed the low-temperature transitions of 1,1'- and 3,1'-DMBMPs contain two or three peaks, indicating the transition took place in two or three steps, although X-ray measurements failed to detect the details of the structural origin of the transitions. Furthermore, we found that the membranes of these lipids exhibit a transition from a bound to an unbound state, coinciding with the $\mathrm{L}_{\mathrm{c}}$ to $\mathrm{L}_{\alpha}$ main phase transition. As shown in Figure 3, the diffraction peaks that originated from multilamellar structure disappeared in the temperature range of $35-40{ }^{\circ} \mathrm{C}$, where the main phase transition starts to occur. The sudden disappearance of the lamellar peaks and the simultaneous appearance of diffuse scattering from the bilayer form factor indicate that the multilamellar membranes were extensively swollen and the defined intermembrane correlation $(\sim 5.2$ $\mathrm{nm})$ was totally lost over a very narrow range of temperatures, i.e., unbound transition $(56-60)$. Figure 5 shows the strong reversibility of this transition of DMBMPs. PozoNavas et al. first observed an unbinding transition in charged MLVs composed of 1-palmitoyl-2-oleoylphosphatidylethanolamine (POPE) and 1-palmitoyl-2-oleoylphosphatidylglycerol (POPG) with small- and wide-angle X-ray scattering (60). They concluded that the unbinding transition of the charged membrane is a direct result of the steric repulsion caused by enhanced thermal undulations of the bilayers in the $\mathrm{L}_{\alpha}$ phase and not a result of electrostatic double-layer repulsion, since no unbinding was observed in the pure DPPG system for a series of differing salt concentrations.

In this study, DMBMPs exhibited their main phase transition at $40-42{ }^{\circ} \mathrm{C}$, which is much higher than that of DMPC and DMPG $\left(\sim 23^{\circ} \mathrm{C}\right)$. These results taken from DSC and X-ray, together with the previous studies on DMPG (43-
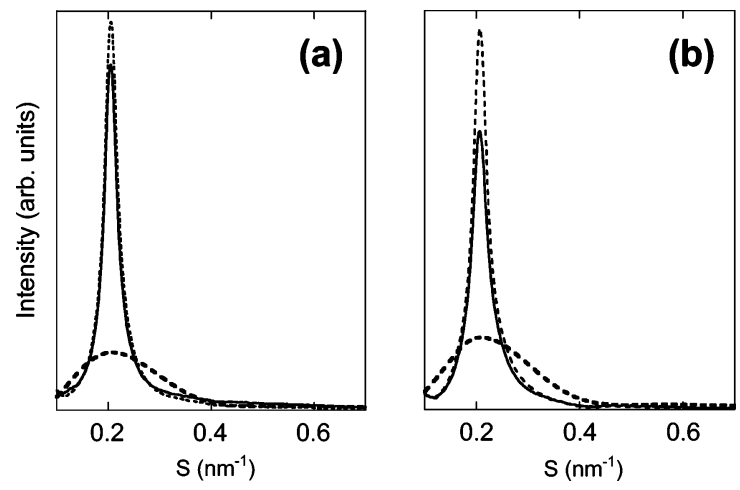

FIGURE 5: Reversible unbinding transition upon heating through the main transition of (a) 1,1'- and (b) 3,1'-DMBMP. Small-angle scattering profiles were taken at $10(-)$ or $45^{\circ} \mathrm{C} \mathrm{(--} \mathrm{-)} \mathrm{on} \mathrm{heating}$ and at $10{ }^{\circ} \mathrm{C} \mathrm{(-} \mathrm{-)} \mathrm{after} \mathrm{cooling.}$

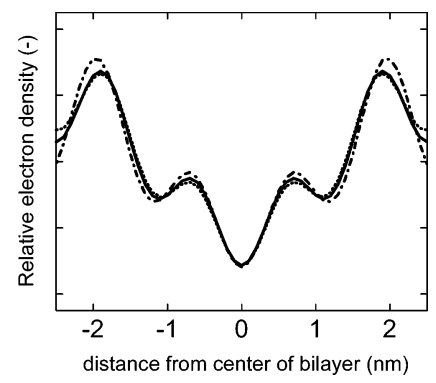

FIGURE 6: Electron density profiles of $1,1^{\prime}$-DMBMP at $5(-)$ and $20{ }^{\circ} \mathrm{C}(-\bullet)$, calculated from the small-angle X-ray data shown in Figure 5. The electron density profile of $3,1^{\prime}$-DMBMP at $5{ }^{\circ} \mathrm{C}$ (- -) is also shown. The geometric center of the bilayer is at the origin.

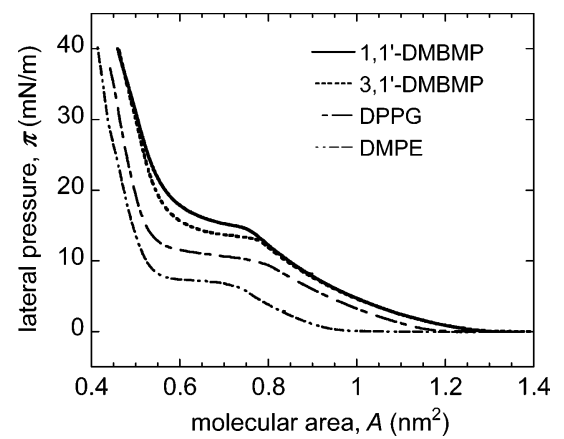

FIGURE 7: Surface pressure $(\pi)$ vs mean molecular area $(A)$ isotherms for $1,1^{\prime}$-DMBMP, 3, $1^{\prime}$-DMBMP, DPPG, and DMPE obtained at room temperature $\left(23.5 \pm 0.5{ }^{\circ} \mathrm{C}\right)$ on a subphase of HEPES buffer containing $100 \mathrm{mM} \mathrm{NaCl}$ and $10 \mathrm{mM}$ EDTA $(\mathrm{pH}$ 7.0). All lipid films were compressed at a rate of $0.1 \mathrm{~nm}^{2}$ per molecule per minute.

48), suggest the presence of a strong attractive interaction between the headgroups of DMBMP. Intermolecular hydrogen bonding via the $\mathrm{PO}^{-}$group, the glycerol $\mathrm{OH}$ group, and the fatty acid $\mathrm{C}=\mathrm{O}$ group would be the most probable candidates for this attractive force. These attractive interactions between the headgroups and the interface region may be responsible for the packing of lipids in an ordered lattice at low temperatures, and a main phase transition takes place at high temperatures with a large transition enthalpy. A sudden decrease in the membrane rigidity can occur when the thermal motion of the lipids overcomes the intermolecular hydrogen bonding at the main transition, resulting in the cooperative unbinding of DMBMP membranes. 

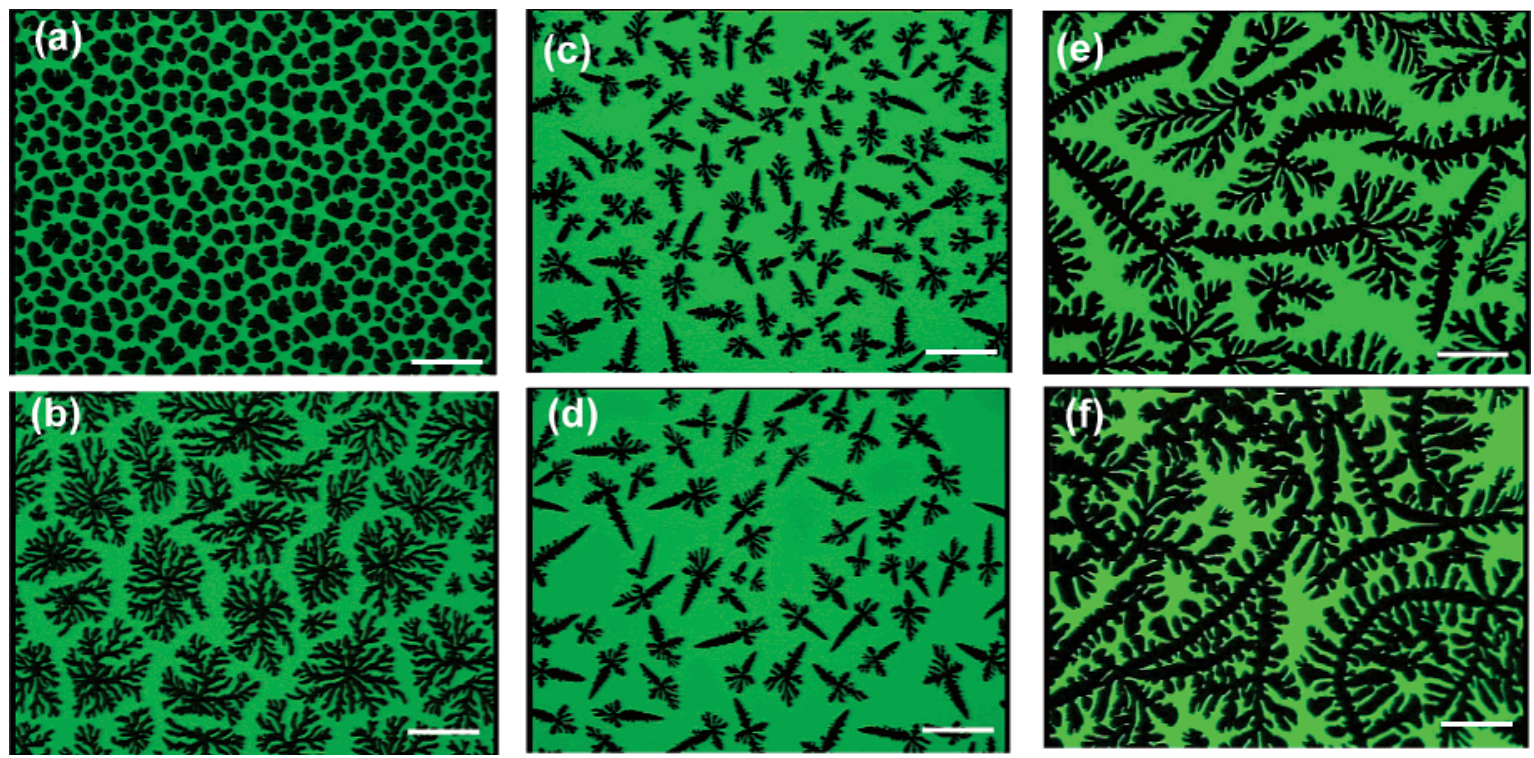

FIGURE 8: Fluorescence micrographs of lipid monolayers in the liquid-expanded and liquid-condensed phase coexistence region, observed after fast compression: (a) DPPG, (b) DMPE, (c) 1,1'-DMBMP, and (d) 3,1'-DMBMP. In all cases, the same subphase condition was employed, $20 \mathrm{mM}$ HEPES buffer containing $100 \mathrm{mM} \mathrm{NaCl}$ and $10 \mathrm{mM}$ EDTA (pH 7.0). Panels a-d were taken around the $\pi_{\mathrm{c}}$ of the respective lipid. Panels e and $\mathrm{f}$ are fluorescence micrographs of $1,1^{\prime}$-DMBMP and $3,1^{\prime}$-DMBMP, taken above the $\pi_{\mathrm{c}}$. Scale bars are 100 $\mu \mathrm{m}$. The fluorescent probe (Bodipy- $\mathrm{C}_{12}-\mathrm{PC}$ ) segregates in the disordered or fluid phases, which then appear bright in the images.

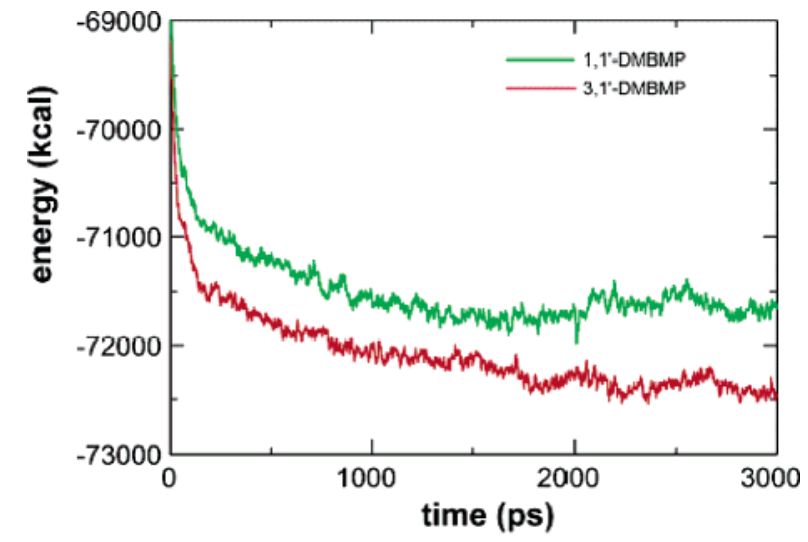

FIGURE 9: Energy profiles of 1,1'- (green) and 3,1'-DMBMP (red) up to 3 ns.

Electron Density Profiles of DMBMP. We then calculated the electron density distribution for the 1,1'-DMBMP membrane at 5 and $20{ }^{\circ} \mathrm{C}$ (Figure 6). In this figure, the geometric center of the bilayer is at the origin. The lowelectron density region in the center of the profile corresponds to the lipid hydrocarbon chains. The high-density regions, located within $\sim 2 \mathrm{~nm}$ of the bilayer center, correspond mainly to the phosphate groups of $1,1^{\prime}$-DMBMP. Although these two profiles at 5 and $20^{\circ} \mathrm{C}$ exhibit a similar pattern in the hydrophobic region, a slight difference was observed in the peak position, i.e., a peak at $\pm 1.90 \mathrm{~nm}$ for $5{ }^{\circ} \mathrm{C}$ and at $\pm 1.97 \mathrm{~nm}$ for $20^{\circ} \mathrm{C}$. This result indicates that the observed increase in the lamellar repeat distance from 5.04 to 5.22 nm shown in Figure 3 can mainly be attributed to the increase in the membrane width. Considering the observations via SAXS, WAXS, and DSC, the increase in the membrane width corresponds to the change in the tilt angle of the acyl chains. The electron density profile for 3,1'-DMBMP was also calculated with the same phase combination that was used for 1,1'-DMBMP. As shown in Figure 6, although the overall profile was quite similar to that of $1,1^{\prime}$-DMBMP, the distance between the phosphate peaks in 3,1'-DMBMP was slightly shorter than that in $1,1^{\prime}$-DMBMP, as expected.

Pressure-Area Compression Isotherms and Epifluorescence Microscopy. The behavior of DMBMPs at the airbuffer interface was investigated by using pressure - area $(\pi-$ $A$ ) isotherms and epifluorescence microscopy. Figure 7 shows the surface pressure versus mean molecular area isotherms of 1,1'-DMBMP, 3,1'-DMBMP, DMPE, and DPPG at $\mathrm{pH}$ 7.0. For all lipids, the coexisting regions of both the condensed and expanded phases were observed at various pressures. At $30 \mathrm{mN} / \mathrm{m}$, the areas per molecule observed for DMPE and DPPG were 0.43 and $0.46 \mathrm{~nm}^{2}$, respectively. These values are fairly consistent with the previous results obtained under similar conditions $(61-63)$. 1, $1^{\prime}-$ and $3,1^{\prime}$ DMBMP gave an area per molecule of 0.50 and $0.49 \mathrm{~nm}^{2}$, respectively. These DMBMP values are larger than those of the published results of DMPA, DMPE, DPPC, and DPPG (61-64). DMPG, a structural isomer of DMBMP, did not show any phase transition under these conditions because its melted hydrocarbon chains could not pack densely and remained disordered, although it does exhibit a condensed phase under certain conditions (65). The reported area per molecule for DMPG at $5{ }^{\circ} \mathrm{C}$ and $\mathrm{pH} 7$ in the presence of $100 \mathrm{mM} \mathrm{NaCl}$ is $\sim 0.59 \mathrm{~nm}^{2}(65)$. The pressure value at the main transition $\left(\pi_{\mathrm{c}}\right)$, where the condensed phase starts to appear, was higher for 1,1'-DMBMP than for 3,1'-DMBMP. This may be caused by the lower phase transition temperature of $1,1^{\prime}$-DMBMP, because the increase in $\pi_{\mathrm{c}}$ can be attributed to the increased level of thermal motion, which tends to expand the lipid membrane, implying a weaker intermolecular interaction. The following order of $\pi_{\mathrm{c}}$ values for different lipids was found: 1,1'-DMBMP > 3,1'-DMBMP > DPPG $>$ DMPE. Under similar solvent conditions, it is reported the main phase transition temperatures of DPPG and DMPE as determined by DSC are $\sim 41.3{ }^{\circ} \mathrm{C}(44,53,66)$ and $>49.5$ ${ }^{\circ} \mathrm{C}(40,67,68)$, respectively. Therefore, the order of $\pi_{\mathrm{c}}$ values 
(a)

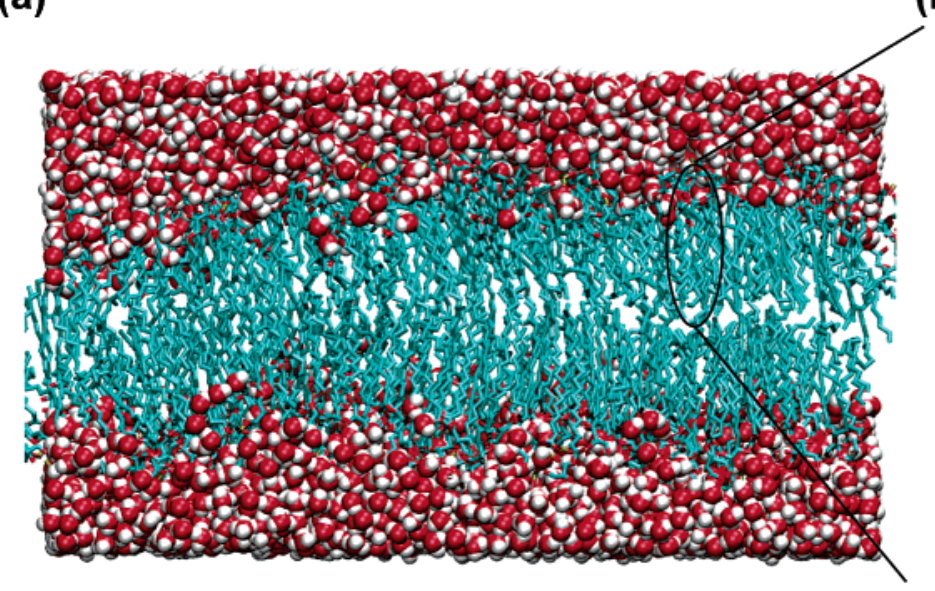

(b)

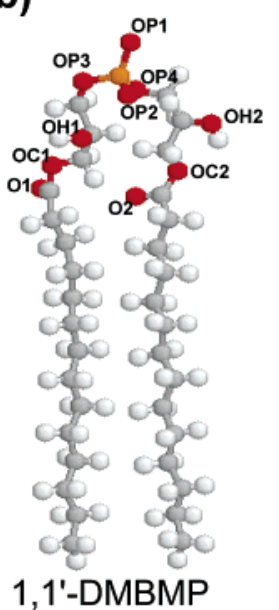

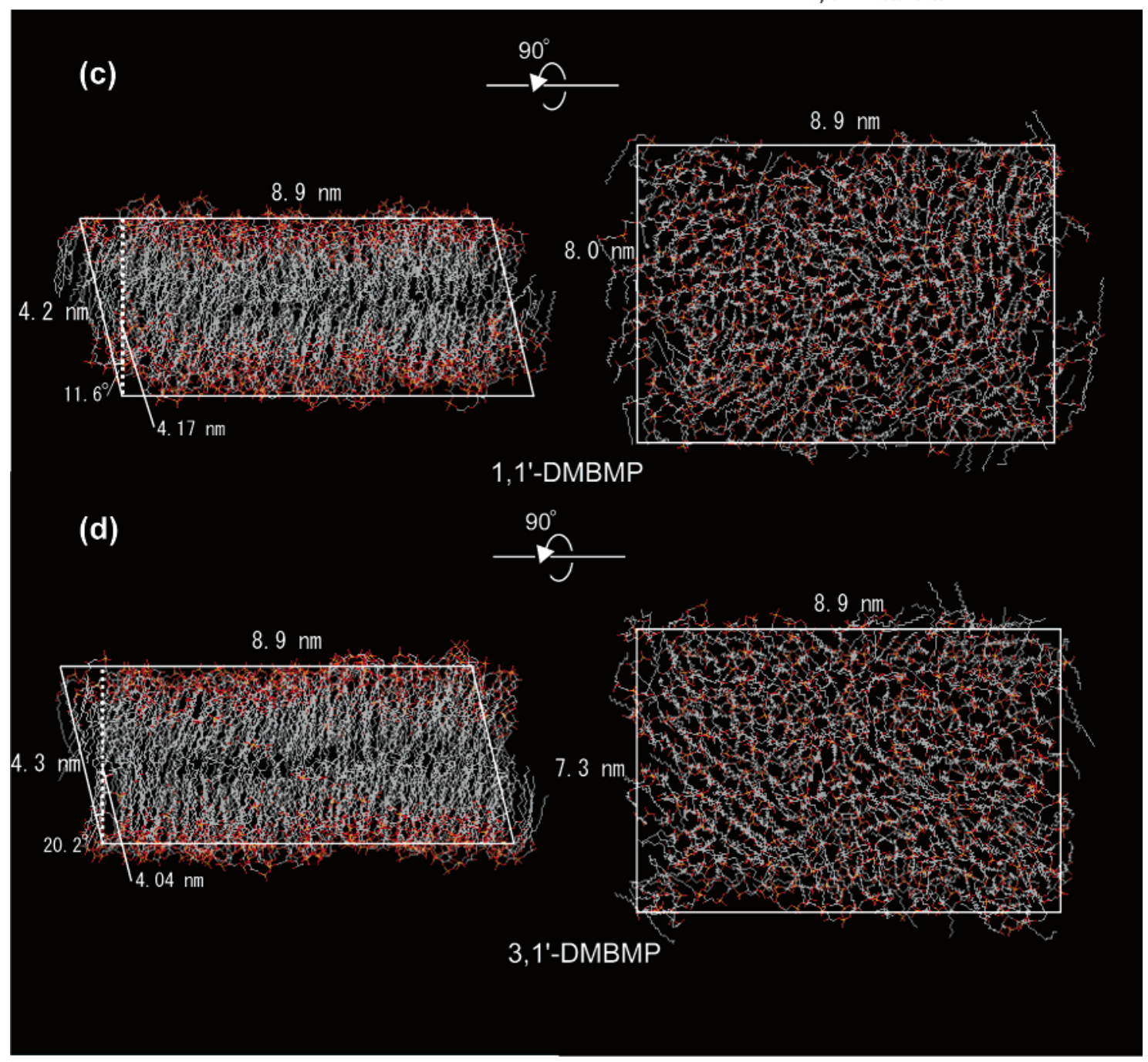

Figure 10: Snapshots of the 1,1'-DMBMP bilayer system at $3.0 \mathrm{~ns}$ (a) and a molecular model of 1,1'-DMBMP (b). Snapshots of (c) $1,1^{\prime}$ and (d) 3,1'-DMBMP bilayer systems which displayed neither hydrogen atoms nor water molecules.

given above reflects, at least in part, the order of the main phase transition temperatures of these lipids.

We then investigated the domain formation of the BMPs at the air-buffer interface to evaluate the intermolecular interactions and the orientational ordering of the headgroups in comparison with that of DMPE and DPPG. Panels $a-d$ of Figure 8 show the fluorescence micrographs of the DPPG, DMPE, 1,1'-DMBMP, and 3,1'-DMBMP monolayers, respectively, in the liquid-expanded and liquid-condensed coexistence region. The fluorescent probe (Bodipy- $\mathrm{C}_{12}-\mathrm{PC}$ ) segregates in the disordered or fluid phases, which then appear bright in the images (69). The probe is absent from the solid or liquid condensed phases, which appear black. Under these conditions, DPPG exhibits a cardioid domain at $11 \mathrm{mN} / \mathrm{m}$, while DMPE exhibits a fractal-like or dendrite domain. The domain shape of DMPE is very similar to that of previous reports $(61,70-72)$. Both DMBMPs displayed a characteristic elongated dendritic domain at $\pi_{\mathrm{c}}(13-15 \mathrm{mN} /$ 
(a)

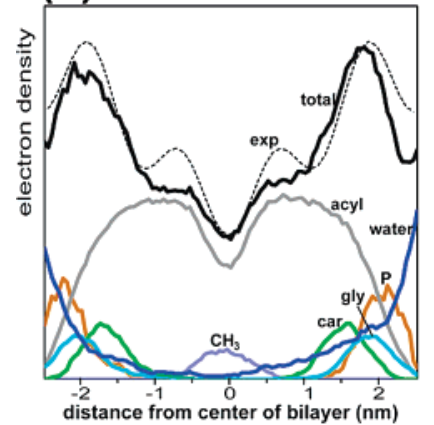

FIGURE 11: Electron density profiles (total) of (a) 1,1'- and (b) $3,1^{\prime}$-DMBMP at $3.0 \mathrm{~ns}$ are shown with the one from X-ray data. Electron density profiles of respective functional groups are also shown in the figure $(\mathrm{P}$, phosphate group; gly, glycerol moiety; car, carbonyl group; $\mathrm{CH}_{3}$, methyl group; acyl, acyl chain).

$\mathrm{m})$. These elongated domains grew as the surface pressure increased (Figure 8e,f). To the best of our knowledge, such anisotropic domains have not been described for naturally occurring lipids, even in the nonequilibrium state. This anisotropic dendritic domain shape is indicative of the formation of an orientationally ordered solid phase. It is known that the orientational order and chirality of lipid headgroups in the monolayers at the air-water interface are reflected in the domain shape of the lipid $(61,72-74)$. Domain shapes are thought to be determined by a competition between line tension (that tends to stabilize isotropic circular domains) and long-range dipole-dipole repulsion between lipid molecules (that tends to stabilize elongated domains) (75). Thus, charged lipids generally tend to form elongated domains. Under these conditions, the 1,1'- and 3,1'DMBMP domains exhibit a similar shape, suggesting both DMBMPs are packed in a similar manner in the monolayers at the air-buffer interface.

Molecular Dynamics (MD) Simulations. We performed a total of $6 \mathrm{~ns}$ MD simulations of 1,1'- and 3,1'-DMBMPs to investigate the intermolecular interactions and dynamics of DMBMPs. To determine whether the MD simulations reached equilibrium states, the energies of $1,1^{\prime}-$ and $3,1^{\prime}$ DMBMPs during simulations were calculated. Figure 9 shows that the MD simulations reached equilibrium states after $2.0 \mathrm{~ns}$. Therefore, the data collection for the analysis of each DMBMP was to last 1.0 ns. Figure 10 shows the snapshots of the 1,1'-DMBMP bilayer system (a) and a $1,1^{\prime}$ DMBMP molecule (b) at $3.0 \mathrm{~ns}$. To compare the membrane structure obtained by MD simulation with the one from the $\mathrm{X}$-ray experiment, the electron density maps from MD simulations were calculated (Figure 11). The electron density map for the 1,1'- and 3,1'-DMBMPs resembles the experimental data (dotted lines in Figure 11). This indicates that the membrane and molecular structure of DMBMP obtained from MD simulations reproduce well the experimental findings. The thickness of the membrane (distances between the $\mathrm{P}$ atoms in headgroups) of $1,1^{\prime}$ - and 3,1'-DMBMPs was 4.17 and $4.04 \mathrm{~nm}$, respectively. The averaged tilt angles of $1,1^{\prime}$ - and 3,1'-DMBMPs to the bilayer normal orientation were 11.6 and $20.2^{\circ}$, respectively. The difference in this tilt angle of the lipids accounts for the difference in the thickness of the membranes. The total system areas of the headgroups of 1,1'- and 3,1'-DMBMPs, which contain 128 molecules, were 71.20 and $64.97 \mathrm{~nm}^{2}$, respectively (Figure 10c,d).

Table 2: Number of Water-Lipid Hydrogen Bonds Formed between Water and the Respective Functional Groups in 1,1'- and 3,1'-DMBMP, Calculated from MD Simulations at $278 \mathrm{~K}^{a}$

\begin{tabular}{lll}
\hline & $3,1^{\prime}$-DMBMP & $1,1^{\prime}$-DMBMP \\
\hline OP1 & $1.996 \pm 0.035$ & $1.703 \pm 0.012$ \\
OP2 & $1.789 \pm 0.063$ & $1.593 \pm 0.051$ \\
OP3 & $0.750 \pm 0.012$ & $0.711 \pm 0.027$ \\
OP4 & $1.016 \pm 0.027$ & $0.980 \pm 0.051$ \\
OH1 & $0.836 \pm 0.035$ & $0.809 \pm 0.008$ \\
OH2 & $0.961 \pm 0.055$ & $0.910 \pm 0.023$ \\
OC1 & $0.422 \pm 0.008$ & $0.398 \pm 0.023$ \\
OC2 & $0.500 \pm 0.004$ & $0.492 \pm 0.004$ \\
O1 & $0.461 \pm 0.008$ & $0.430 \pm 0.020$ \\
O2 & $0.461 \pm 0.004$ & $0.398 \pm 0.004$ \\
total & $9.192 \pm 0.025$ & $8.404 \pm 0.022$ \\
\hline
\end{tabular}

${ }^{a}$ The assignments of the functional groups (atoms) are as shown in Figure $10 \mathrm{~b}$.

Table 3: Number of Intermolecular Hydrogen Bonds Formed between the $\mathrm{OH}$ Group of the Glycerol Moiety in 1,1'- and 3,1'-DMBMP and Functional Groups of the Adjacent Molecule, Calculated from MD Simulations at $278 \mathrm{~K}^{a}$

\begin{tabular}{lccccc}
\hline & \multicolumn{2}{c}{$3,1^{\prime}$-DMBMP } & & \multicolumn{2}{c}{$1,1^{\prime}$-DMBMP } \\
\cline { 2 - 3 } \cline { 5 - 6 } & OH1 & OH2 & & OH1 & OH2 \\
\hline OP1 & $0.046 \pm 0.002$ & $0.048 \pm 0.002$ & & $0.065 \pm 0.022$ & $0.056 \pm 0.007$ \\
OP2 & $0.063 \pm 0.004$ & $0.069 \pm 0.011$ & & $0.048 \pm 0.001$ & $0.086 \pm 0.011$ \\
OP3 & $0.042 \pm 0.007$ & $0.060 \pm 0.027$ & & $0.077 \pm 0.002$ & $0.054 \pm 0.020$ \\
OP4 & $0.028 \pm 0.007$ & $0.026 \pm 0.003$ & & $0.025 \pm 0.005$ & $0.023 \pm 0.001$ \\
OH1 & $0.058 \pm 0.003$ & $0.102 \pm 0.043$ & & $0.093 \pm 0.005$ & $0.088 \pm 0.010$ \\
OH2 & $0.090 \pm 0.011$ & $0.039 \pm 0.004$ & & $0.088 \pm 0.010$ & $0.093 \pm 0.005$ \\
OC1 & $0.048 \pm 0.002$ & $0.121 \pm 0.027$ & & $0.073 \pm 0.030$ & $0.088 \pm 0.001$ \\
OC2 & $0.122 \pm 0.021$ & $0.049 \pm 0.002$ & & $0.074 \pm 0.012$ & $0.038 \pm 0.005$ \\
O1 & $0.086 \pm 0.001$ & $0.064 \pm 0.010$ & & $0.076 \pm 0.002$ & $0.094 \pm 0.003$ \\
O2 & $0.081 \pm 0.001$ & $0.129 \pm 0.007$ & & $0.044 \pm 0.002$ & $0.082 \pm 0.027$ \\
total & $0.688 \pm 0.000$ & $0.706 \pm 0.049$ & & $0.664 \pm 0.027$ & $0.640 \pm 0.075$ \\
\hline
\end{tabular}

${ }^{a}$ The assignments of the functional groups (atoms) are as shown in Figure $10 \mathrm{~b}$

Therefore, the average single molecular area of the DMBMPs is simply calculated by dividing these values by 128 , that is, $\sim 0.56 \mathrm{~nm}^{2}$ for $1,1^{\prime}$-DMBMP and $\sim 0.51 \mathrm{~nm}^{2}$ for $3,1^{\prime}$ DMBMP. We then investigated the intermolecular interactions (water-lipid and lipid-lipid hydrogen bonds) in 1,1'and 3,1'-DMBMPs. The average number of lipid-lipid and water-lipid interactions (hydrogen bonds) of 3,1'-DMBMP was slightly larger than that of 1,1'-DMBMP (Tables 2 and $3)$. Thus, these numbers can explain the difference in the phase transition temperature and molecular area between $1,1^{\prime}$ and 3,1'-DMBMP observed in the experiments.

\section{CONCLUDING REMARKS}

This study characterized the thermotropic phase behavior and membrane structure of 1,1'-DMBMP, together with a $3,1^{\prime}$ isomer, by using DSC, X-ray scattering, $\pi-A$ isotherms, epifluorescence microscopy of the lipid monolayer, and MD simulations. DSC, X-ray scattering, and MD simulation showed that these DMBMPs are packed in a quasi-crystalline state in the membrane below the main phase transition temperature. At the main phase transition, both DMBMPs undergo a quasi-crystalline to $\mathrm{L}_{\alpha}$ transition accompanying unbinding of the membranes; that is, the stacked lamellar membranes transform from a bound to an unbound state. The main transition temperature and the enthalpy value observed at $\mathrm{pH} 7.0$ were $40.1^{\circ} \mathrm{C}$ and $12.4 \mathrm{kcal} / \mathrm{mol}$ for $1,1^{\prime}$ - 
DMBMP and $42.1^{\circ} \mathrm{C}$ and $13.6 \mathrm{kcal} / \mathrm{mol}$ for $3,1^{\prime}$-DMBMP, respectively. These values are much higher than those of DMPG, a structural isomer and a potential precursor of DMBMP. MD simulations revealed both DMBMPs form a compact conformation in the membrane such that the headgroup is bent to arrange the two acyl chains close to each other and the $\mathrm{OH}$ groups of the glycerol moieties face adjacent molecules (Figure 10). MD simulations also indicated that the $\mathrm{OH}$ groups of the glycerol moieties can interact with the $\mathrm{PO}^{-}$and $\mathrm{C}=\mathrm{O}$ groups of adjacent molecules via hydrogen bonding. The capacity for hydrogen bond formation was greater for $3,1^{\prime}$-DMBMP than for $1,1^{\prime}$-DMBMP. This explains the higher phase transition temperature of $3,1^{\prime}$ DMBMP. This difference can be attributed to the structural differences in the headgroup, since the two $\mathrm{OH}$ groups of $3,1^{\prime}$-DMBMP are bilaterally symmetric and oriented toward adjacent molecules, whereas those of $1,1^{\prime}$-DMBMP are asymmetric. In addition, the glycerol moiety of the 3,1'DMBMP headgroup has a tendency for ordered lattice formation via a hydrogen bonding network in the membrane. Such a difference, depending on the stereoconfiguration, is also reported for 1,2-dimyristoyl-sn-glycero-3-phosphatidyl$s n-1^{\prime}$-glycerol (1'-DMPG) and the corresponding $s n-3^{\prime}$ stereoisomer (3'-DMPG). The 3'-DMPG- $\mathrm{Na}^{+}$complex forms an ordered, highly crystalline phase, which has a transition into the liquid crystalline phase at a temperature higher than that of the $1^{\prime}$-DMPG $-\mathrm{Na}^{+}$complex. The higher affinity of $3^{\prime}$-DMPG for $\mathrm{Na}^{+}$is suggested to be due to a slightly different favored conformation of the headgroup glycerol moiety $(48-50)$.

Whereas 3,3'- and 1,3'-diacyl-BMP are thermodynamically stable, a recent study (5) suggests that in cells, 2,2'-diacylBMP is dominant. Thus, 3,3'-diacyl-BMP is physically stable but biologically unstable. There has been speculation that there is a dynamic equilibrium between $2,2^{\prime}$ and $3,3^{\prime}$ isomers inside late endosomes. The role of $\mathrm{OH}$ groups in physically unstable 2,2'-diacyl-BMP is the next challenging subject.

\section{ACKNOWLEDGMENT}

We are grateful to K. Iwamoto, H. Shogomori, R. Ishitsuka, M. Murate, K. Ishii, Y. Ueda, and K. Shibata for their technical help in SAXS measurements at SPring-8. We thank K. Iwamoto, H. Shogomori, R. Ishitsuka, and F. HullinMatsuda for critically reading the manuscript.

\section{REFERENCES}

1. Body, D., and Gray, G. (1967) The isolation and characterization of phosphatidylglycerol and a structural isomer from pig lung, Chem. Phys. Lipids 1, 254-63.

2. Rouser, G., and Solomon, R. D. (1969) Changes in phospholipid composition of human aorta with age, Lipids 4, 232-4.

3. Mason, R. J., Stossel, T. P., and Vaughan, M. (1972) Lipids of alveolar macrophages, polymorphonuclear leukocytes, and their phagocytic vesicles, J. Clin. Invest. 51, 2399-407.

4. Kobayashi, T., Stang, E., Fang, K. S., de Moerloose, P., Parton, R. G., and Gruenberg, J. (1998) A lipid associated with the antiphospholipid syndrome regulates endosome structure and function, Nature 392, 193-7.

5. Kobayashi, T., Beuchat, M. H., Chevallier, J., Makino, A., Mayran, N., Escola, J. M., Lebrand, C., Cosson, P., Kobayashi, T., and Gruenberg, J. (2002) Separation and characterization of late endosomal membrane domains, J. Biol. Chem. 277, 32157-64.
6. Le Blanc, I., Luyet, P. P., Pons, V., Ferguson, C., Emans, N., Petiot, A., Mayran, N., Demaurex, N., Faure, J., Sadoul, R., Parton, R. G., and Gruenberg, J. (2005) Endosome-to-cytosol transport of viral nucleocapsids, Nat. Cell Biol. 7, 653-64.

7. Kobayashi, T., Beuchat, M. H., Lindsay, M., Frias, S., Palmiter, R. D., Sakuraba, H., Parton, R. G., and Gruenberg, J. (1999) Late endosomal membranes rich in lysobisphosphatidic acid regulate cholesterol transport, Nat. Cell Biol. 1, 113-8.

8. Wilkening, G., Linke, T., Uhlhorn-Dierks, G., and Sandhoff, K. (2000) Degradation of membrane-bound ganglioside GM1. Stimulation by bis(monoacylglycero)phosphate and the activator proteins SAP-B and GM2-AP, J. Biol. Chem. 275, 35814-9.

9. Werth, N., Schuette, C. G., Wilkening, G., Lemm, T., and Sandhoff, K. (2001) Degradation of membrane-bound ganglioside GM2 by $\beta$-hexosaminidase A. Stimulation by GM2 activator protein and lysosomal lipids, J. Biol. Chem. 276, 12685-90.

10. Linke, T., Wilkening, G., Sadeghlar, F., Mozcall, H., Bernardo, K., Schuchman, E., and Sandhoff, K. (2001) Interfacial regulation of acid ceramidase activity. Stimulation of ceramide degradation by lysosomal lipids and sphingolipid activator proteins, J. Biol. Chem. 276, 5760-8.

11. Brotherus, J., Renkonen, O., Fischer, W., and Herrmann, J. (1974) Novel stereoconfiguration in lyso-bis-phosphatidic acid of cultured BHK-cells, Chem. Phys. Lipids 13, 178-82.

12. Joutti, A., Brotherus, J., Renkonen, O., Laine, R., and Fischer, W. (1976) The stereochemical configuration of lysobisphosphatidic acid from rat liver, rabbit lung and pig lung, Biochim. Biophys. Acta 450, 206-9.

13. Joutti, A., and Renkonen, O. (1979) The stereoconfiguration of bis(monoacylglycero)phosphate synthesized in vitro in lysosomes of rat liver: Comparison with the natural lipid, J. Lipid Res. 20, $840-7$.

14. Joutti, A. (1979) The stereoconfiguration of newly formed molecules of bis(monoacylglycero)phosphate in BHK cells, Biochim. Biophys. Acta 575, 10-5.

15. Heravi, J., and Waite, M. (1999) Transacylase formation of bis(monoacylglycerol)phosphate, Biochim. Biophys. Acta 1437, 27786.

16. Amidon, B., Schmitt, J. D., Thuren, T., King, L., and Waite, M. (1995) Biosynthetic conversion of phosphatidylglycerol to sn-1: sn-1' bis(monoacylglycerol) phosphate in a macrophage-like cell line, Biochemistry 34, 5554-60.

17. Amidon, B., Brown, A., and Waite, M. (1996) Transacylase and phospholipases in the synthesis of bis(monoacylglycero)phosphate, Biochemistry 35, 13995-4002.

18. Thornburg, T., Miller, C., Thuren, T., King, L., and Waite, M. (1991) Glycerol reorientation during the conversion of phosphatidylglycerol to bis(monoacylglycerol)phosphate in macrophagelike RAW 264.7 cells, J. Biol. Chem. 266, 6834-40.

19. Waite, M., Roddick, V., Thornburg, T., King, L., and Cochran, F. (1987) Conversion of phosphatidylglycerol to lyso(bis)phosphatidic acid by alveolar macrophages, FASEB J. 1, 318-25.

20. Waite, M., King, L., Thornburg, T., Osthoff, G., and Thuren, T. Y. (1990) Metabolism of phosphatidylglycerol and bis(monoacylglycero)-phosphate in macrophage subcellular fractions, $J$. Biol. Chem. 265, 21720-6.

21. Somerharju, P., and Renkonen, O. (1980) Conversion of phosphatidylglycerol lipids to bis(monoacylglycero)phosphate in vivo, Biochim. Biophys. Acta 618, 407-19.

22. Ito, M., Tchoua, U., Okamoto, M., and Tojo, H. (2002) Purification and properties of a phospholipase A2/lipase preferring phosphatidic acid, bis(monoacylglycerol) phosphate, and monoacylglycerol from rat testis, J. Biol. Chem. 277, 43674-81.

23. Holopainen, J. M., Soderlund, T., Alakoskela, J. M., Saily, M., Eriksson, O., and Kinnunen, P. K. (2005) Intermolecular interactions of lysobisphosphatidic acid with phosphatidylcholine in mixed bilayers, Chem. Phys. Lipids 133, 51-67.

24. Matsuo, H., Chevallier, J., Mayran, N., Le Blanc, I., Ferguson, C., Faure, J., Blanc, N. S., Matile, S., Dubochet, J., Sadoul, R., Parton, R. G., Vilbois, F., and Gruenberg, J. (2004) Role of LBPA and Alix in multivesicular liposome formation and endosome organization, Science 303, 531-4.

25. Makino, A., Ishii, K., Murate, M., Hayakawa, T., Suzuki, Y., Suzuki, M., Ito, K., Fujisawa, T., Matsuo, H., Ishitsuka, R., and Kobayashi, T. (2006) D-threo-1-Phenyl-2-decanoylamino-3-morpholino-1-propanol alters cellular cholesterol homeostasis by modulating the endosome lipid domains, Biochemistry 45, 453041. 
26. Chevallier, J., Sakai, N., Robert, F., Kobayashi, T., Gruenberg, J., and Matile, S. (2000) Rapid access to synthetic lysobisphosphatidic acids using P(III) chemistry, Org. Lett. 2, 1859-61.

27. Garrigues, B., Bertrand, G., Frehel, D., and Maffrand, J. (1984) Synthesis of d,1-[2-acetoxy-1-(octadecylthio)]-3-propylphosphorylcholine or thia-PAF, Phosphorus Sulfur 21, 171-6.

28. Fujisawa, T., Inoue, K., Oka, T., Iwamoto, H., Uraga, T., Kumasaka, T., Inoko, Y., Yagi, N., Yamamoto, M., and Ueki, T. (2000) Small-angle X-ray scattering station at the SPring-8 RIKEN beamline, J. Appl. Crystallogr. 33, 797-800.

29. Cherezov, V., Riedl, K. M., and Caffrey, M. (2002) Too hot to handle? Synchrotron X-ray damage of lipid membranes and mesophases, J. Synchrotron Radiat. 9, 333-41.

30. Cheng, A. C., Hogan, J. L., and Caffrey, M. (1993) X-rays destroy the lamellar structure of model membranes, J. Mol. Biol. 229, 291-4.

31. Fujisawa, T., Nishikawa, Y., Yamazaki, H., and Inoko, Y. (2003) Evaluation and improvements of the Rigaku imaging plate reader $\left(\mathrm{R}\right.$-Axis $\mathrm{IV}^{++}$) for the use in synchrotron X-ray solution scattering, J. Appl. Crystallogr. 36, 535-9.

32. Huang, T., Toraya, H., Blanton, T., and Wu, Y. (1993) X-ray powder diffraction analysis of silver behenate, a possible lowangle diffraction standard, J. Appl. Crystallogr. 26, 180-4.

33. Herbette, L., Marquardt, J., Scarpa, A., and Blasie, J. K. (1977) A direct analysis of lamellar X-ray diffraction from hydrated oriented multilayers of fully functional sarcoplasmic reticulum, Biophys. J. 20, 245-72.

34. McIntosh, T. J., and Simon, S. A. (1994) Long- and short-range interactions between phospholipid/ganglioside GM1 bilayers, Biochemistry 33, 10477-86.

35. Frisch, M. J., Trucks, G. W., Schlegel, H. B., Scuseria, G. E., Robb, M. A., Cheeseman, J. R., Montgomery, J. M., Iyengar, S. S., Tomasi, J., Barone, V., Mennucci, B., Cossi, M., Scalmani, G., Rega, N., Petersson, G. A., Nakatsuji, H., Hada, M., Ehara, M., Toyota, K., Fukuda, R., Hasegawa, J., Ishida, M., Nakajima, T., Honda, Y., Kitao, O., Nakai, H., Klene, M., Li, X., Knox, J. E., Hratchian, H. P., Cross, J. B., Adamo, C., Jaramillo, J., Gomperts, R., Stratmann, R. E., Yazyev, O., Austin, A. J., Cammi, R., Pomelli, C., Ochterski, J. W., Ayala, P. Y., Morokuma, K., Voth, G. A., Salvador, P., Dannenberg, J. J., Zakrzewski, V. G., Dapprich, S., Daniels, A. D., Strain, M. C., Farkas, O., Malick, D. K., Rabuck, A. D., Raghavachari, K., Foresman, J. B., Ortiz, J. V., Cui, Q., Baboul, A. G., Clifford, S., Cioslowski, J., Stefanov, B. B., Liu, G., Liashenko, A., Piskorz, P., Komaromi, I., Martin, R. L., Fox, D. J., Keith, T., Al-Laham, M. A., Peng, C. Y., Nanayakkara, A., Challacombe, M., Gill, P. M. W., Johnson, B., Chen, W., Wong, M. W., Gonzalez, C., and Pople, J. A. (2004) Gaussian, revision 03, Gaussian Inc., Wallingford, CT.

36. Case, D. A., Darden, T. A., Cheatham, T. E. I., Simmerling, C. L., Wang, J., Duke, R. E., Luo, R., Merz, K. M., Wang, B., Pearlman, D. A., Crowley, M., Brozell, S., Tsui, V., Gohlke, H., Mongan, J., Hornak, V., Cui, G., Beroza, P., Schafmeister, C., Caldwell, J. W., Ross, W. S., and Kollman, P. A. (2004) Amber, version 8, University of California, San Francisco.

37. van Dijck, P. W., de Kruijff, B., Verkleij, A. J., van Deenen, L. L., and de Gier, J. (1978) Comparative studies on the effects of $\mathrm{pH}$ and $\mathrm{Ca}^{2+}$ on bilayers of various negatively charged phospholipids and their mixtures with phosphatidylcholine, Biochim. Biophys. Acta 512, 84-96.

38. Wilkinson, D. A., and Nagle, J. F. (1981) Dilatometry and calorimetry of saturated phosphatidylethanolamine dispersions, Biochemistry 20, 187-92.

39. Ladbrooke, B. D., and Chapman, D. (1969) Thermal analysis of lipids, proteins and biological membranes. A review and summary of some recent studies, Chem. Phys. Lipids 3, 304-56.

40. Mabrey, S., and Sturtevant, J. M. (1976) Investigation of phase transitions of lipids and lipid mixtures by sensitivity differential scanning calorimetry, Proc. Natl. Acad. Sci. U.S.A. 73, 3862-6.

41. Findlay, E. J., and Barton, P. G. (1978) Phase behavior of synthetic phosphatidylglycerols and binary mixtures with phosphatidylcholines in the presence and absence of calcium ions, Biochemistry $17,2400-5$.

42. Janiak, M. J., Small, D. M., and Shipley, G. G. (1976) Nature of the thermal pretransition of synthetic phospholipids: Dimyristolyland dipalmitoyllecithin, Biochemistry 15, 4575-80.

43. Garidel, P., Richter, W., Rapp, G., and Blume, A. (2001) Structural and morphological investigations of the formation of quasicrystalline phase of 1,2-dimyristoyl-sn-glycero-3-phosphoglycerol (DMPG), Phys. Chem. Chem. Phys. 3, 1504-13.
44. Zhang, Y. P., Lewis, R. N., and McElhaney, R. N. (1997) Calorimetric and spectroscopic studies of the thermotropic phase behavior of the n-saturated 1,2-diacylphosphatidylglycerols, Biophys. J. 72, 779-93.

45. Koynova, R. (1997) Liquid crystalline phase metastability of phosphatidylglycerols, Chem. Phys. Lipids 89, 67-73.

46. Kodama, M., Miyata, T., and Yokoyama, T. (1993) Crystalline cylindrical structures of $\mathrm{Na}^{+}$-bound dimyristoylphosphatidylglycerol as revealed by microcalorimetry and electron microscopy, Biochim. Biophys. Acta 1168, 243-8.

47. Epand, R. M., Gabel, B., Epand, R. F., Sen, A., Hui, S. W., Muga, A., and Surewicz, W. K. (1992) Formation of a new stable phase of phosphatidylglycerols, Biophys. J. 63, 327-32.

48. Salonen, I. S., Eklund, K. K., Virtanen, J. A., and Kinnunen, P. K. (1989) Comparison of the effects of $\mathrm{NaCl}$ on the thermotropic behaviour of sn-1' and sn-3' stereoisomers of 1,2-dimyristoyl-snglycero-3-phosphatidylglycerol, Biochim. Biophys. Acta 982, 20515.

49. Lotta, T. I., Salonen, I. S., Virtanen, J. A., Eklund, K. K., and Kinnunen, P. K. (1988) Fourier transform infrared study of fully hydrated dimyristoylphosphatidylglycerol. Effects of $\mathrm{Na}^{+}$on the sn- $1^{\prime}$ and sn-3' headgroup stereoisomers, Biochemistry 27, $8158-$ 69.

50. Eklund, K. K., Virtanen, J. A., Vuori, K., Patrikainen, J., and Kinnunen, P. K. (1987) Role of the polar head group stereoconfiguration in the cation-induced aggregation of dimyristoylphosphatidylglycerol vesicles, Biochemistry 26, 7542-5.

51. Takahashi, H., Matuoka, S., Kato, S., Ohki, K., and Hatta, I. (1992) Effects of poly(L-lysine) on the structural and thermotropic properties of dipalmitoylphosphatidylglycerol bilayers, Biochim. Biophys. Acta 1110, 29-36.

52. Blaurock, A. E., and McIntosh, T. J. (1986) Structure of the crystalline bilayer in the subgel phase of dipalmitoylphosphatidylglycerol, Biochemistry 25, 299-305.

53. Wilkinson, D. A., and McIntosh, T. J. (1986) A subtransition in a phospholipid with a net charge, dipalmitoylphosphatidylglycerol, Biochemistry 25, 295-8.

54. Ruocco, M., and Shipley, G. (1982) Characterization of the subtransition of hydrated dipalmitoylphosphatidylcholine bilayers, Biochim. Biophys. Acta 691, 309-20.

55. Tenchov, B., Koynova, R., and Rapp, G. (2001) New ordered metastable phases between the gel and subgel phases in hydrated phospholipids, Biophys. J. 80, 1873-90.

56. Lipowsky, R., and Leibler, S. (1986) Unbinding transitions of interacting membranes, Phys. Rev. Lett. 56, 2541-4.

57. Mutz, M., and Helfrich, W. (1989) Unbinding transition of a biological model membrane, Phys. Rev. Lett. 62, 2881-4.

58. Vogel, M., Munster, C., Fenzl, W., and Salditt, T. (2000) Thermal unbinding of highly oriented phospholipid membranes, Phys. Rev. Lett. 84, 390-3.

59. Pabst, G., Katsaras, J., and Raghunathan, V. A. (2002) Enhancement of steric repulsion with temperature in oriented lipid multilayers, Phys. Rev. Lett. 88, 128101.

60. Pozo-Navas, B., Raghunathan, V. A., Katsaras, J., Rappolt, M., Lohner, K., and Pabst, G. (2003) Discontinuous unbinding of lipid multibilayers, Phys. Rev. Lett. 91, 028101.

61. Miller, A., Knoll, W., and Mohwald, H. (1986) Fractal growth of crystalline phospholipid domains in monomolecular layers, Phys. Rev. Lett. 56, 2633-6.

62. Helm, C. A., Tippmann-Krayer, P., Mohwald, H., Als-Nielsen, J., and Kjaer, K. (1991) Phases of phosphatidyl ethanolamine monolayers studied by synchrotron X-ray scattering, Biophys. $J$. 60, 1457-76.

63. Neville, F., Cahuzac, M., Konovalov, O., Ishitsuka, Y., Lee, K. Y., Kuzmenko, I., Kale, G. M., and Gidalevitz, D. (2006) Lipid Headgroup Discrimination by Antimicrobial Peptide LL-37: Insight into Mechanism of Action, Biophys. J. 90, 1275-87.

64. Wu, F., Gericke, A., Flach, C. R., Mealy, T. R., Seaton, B. A., and Mendelsohn, R. (1998) Domain structure and molecular conformation in annexin V/1,2-dimyristoyl-sn-glycero-3-phosphate/ $\mathrm{Ca}^{2+}$ aqueous monolayers: A Brewster angle microscopy/infrared reflection-absorption spectroscopy study, Biophys. J. 74, 327381.

65. Garidel, P., and Blume, A. (2005) 1,2-Dimyristoyl-sn-glycero-3phosphoglycerol (DMPG) monolayers: Influence of temperature, $\mathrm{pH}$, ionic strength and binding of alkaline earth cations, Chem. Phys. Lipids 138, 50-9. 
66. Lis, L. J., McAlister, M., Fuller, N., Rand, R. P., and Parsegian, V. A. (1982) Interactions between neutral phospholipid bilayer membranes, Biophys. J. 37, 657-65.

67. Seddon, J. M., Cevc, G., and Marsh, D. (1983) Calorimetric studies of the gel-fluid $\left(\mathrm{L}_{\beta}-\mathrm{L}_{\alpha}\right)$ and lamellar-inverted hexagonal $\left(\mathrm{L}_{\alpha}-\right.$ $\mathrm{H}_{\text {II }}$ ) phase transitions in dialkyl- and diacylphosphatidylethanolamines, Biochemistry 22, 1280-9.

68. Blume, A. (1983) Apparent molar heat capacities of phospholipids in aqueous dispersion. Effects of chain length and head group structure, Biochemistry 22, 5436-42.

69. Feigenson, G. W., and Buboltz, J. T. (2001) Ternary phase diagram of dipalmitoyl-PC/dilauroyl-PC/cholesterol: Nanoscopic domain formation driven by cholesterol, Biophys. J. 80, 2775-88.

70. Heckl, W. M., Losche, M., Cadenhead, D. A., and Mohwald, H. (1986) Electrostatically induced growth of spiral lipid domains in the presence of cholesterol, Eur. Biophys. J. 14, 11-7.
71. Helm, C. A., Laxhuber, L., Losche, M., and Mohwald, H. (1986) Electrostatic interactions in phospholipid membranes I: Influence of monovalent ions, J. Colloid Polym. Sci. 264, 46-55.

72. Mohwald, H., Dietrich, A., Bohm, C., Brezesinski, G., and Thoma, M. (1995) Domain formation in monolayers, Mol. Membr. Biol. $12,29-38$

73. Weis, R. M., and McConnell, H. M. (1984) Two-dimensional chiral crystals of phospholipid, Nature 310, 47-9.

74. Bohm, C., Mohwald, H., Leiserowitz, L., Als-Nielsen, J., and Kjaer, K. (1993) Influence of chirality on the structure of phospholipid monolayers, Biophys. J. 64, 553-9.

75. McConnell, H. M., and Moy, V. T. (1988) Shape of finite twodimensional lipid domains, J. Phys. Chem. 92, 4520-5.

BI060722O 